\title{
Multinational, database cohort study to study mortality and risk factors of mortality in patients with asthma
}

\author{
Katia M.C. Verhamme ${ }^{1}$, Marjolein Engelkes ${ }^{1}$, Maria de Ridder ${ }^{1}$, Elisabeth Svensson ${ }^{2}$, Klara \\ Berencsi ${ }^{2}$, Daniel Prieto-Alhambra ${ }^{3,12}$, Francesco Lapi ${ }^{4}$, Carlo Giaquinto ${ }^{5}$, Gino Picelli ${ }^{5}$, Nada \\ Boudiaf ${ }^{6}$, Frank Albers ${ }^{7}$, Sarah Cockle ${ }^{8}$, Eric Bradford ${ }^{9}$, Robert Suruki ${ }^{10}$, Hana Muellerova ${ }^{11}$, Peter \\ Rijnbeek $^{1}$ and Miriam Sturkenboom ${ }^{1}$ \\ 1Medical Informatics, ErasmusMC, Rotterdam, Netherlands \\ 2Department of Clinical Epidemiology, Aarhus University, Aarhus, Denmark \\ 3Idiap Jordi Gol Primary Care Research Institute, Universitat Autònoma de Barcelona, Barcelona, \\ Spain \\ 4HSD, Italian College of General Practitioners and Primary Care, Firenze, Italy \\ 5Pedianet, Pedianet, Padova, Italy \\ 6Observational Data Analytics, GSK, Uxbridge, United Kingdom \\ 7Respiratory Franchise, GSK, Research Triangle Park, NCUnited States \\ 8Value Evidence \& Outcomes, GSK, Brentford, United Kingdom \\ 9Clinical Development, GSK, Research Triangle Park, NCUnited States \\ 10Respiratory Epidemiology, GSK - formely employed, Research Triangle Park, NCUnited States \\ 11Respiratory Epidemiology, GSK, Uxbridge, United Kingdom \\ 12Musculoskeletal Pharmaco- and Device Epidemiology, University of Oxford, Oxford, United \\ Kingdom
}

Background There is sparse real world evidence on mortality rates (MRs) and risk factors (RF) of mortality in patients with asthma.

Aims To assess all-cause MRs and associated RF in patients with asthma.

Methods Asthma patients, aged $\geq 5$ yrs and with $\geq 1$ yr of follow-up were identified in six EHR databases from the Netherlands (IPCI), Italy (HSD and Pedianet), UK (CPRD), Denmark (AUH) and Spain (SIDIAP). MRs were calculated by dividing the number of deaths by the total person-years (PY). The study period was from 2008-2013. RF of mortality were studied in a subcohort of adult incident asthmatics using Cox regression analysis. Potential RF were age, gender, severe asthma (high dose ICS+controller therapy for at least 120 days), severe asthma exacerbations, smoking and COPD.

Results The cohort included 855,806 asthmatics (mean age 33.5-46.6 yrs; Pedianet: 7.2 yrs). $24,537(2.8 \%)$ patients died resulting in an all-cause MR from 6.0 to $12.8 / 1,000 P Y$. The 
incident asthma cohort included 166,618 patients. In univariate analyses, age, severe asthma and COPD were RF of death. Previous asthma exacerbation was associated with death except in $A U H$. In the multivariate analysis, age $\left(H R_{a d j} 1.1\right)$, female gender $\left(H R_{a d j} 0.6-\right.$ $0.8)$ and previous exacerbations remained associated with death $\left(H R_{\text {adj }} 1.4-2.6\right.$ in first 30 days and $H R_{\text {adj }}$ 1.2-1.5 in $>365$ days after exacerbation). COPD increased the risk of mortality up to $93 \%$ in IPCI, AUH, CPRD and SIDIAP (HRadj 1.3-1.9).

Conclusions Patients with a history of asthma exacerbations, increasing age and COPD have an increased risk of mortality. Asthma control is important, not only to prevent exacerbations but also to prevent mortality. 